OPEN ACCESS

Edited by:

Debbie Guest,

Animal Health Trust, United Kingdom

Reviewed by:

Daniela Proverbio, University of Milan, Italy

Maria Fahie,

Western University of Health

Sciences, United States

${ }^{*}$ Correspondence:

Jonathan T. Ferrar

jonathan.t.ferrari@gmail.com

Specialty section: This article was submitted to Veterinary Regenerative Medicine,

a section of the journal

Frontiers in Veterinary Science

Received: 10 March 2020

Accepted: 11 May 2020

Published: 12 June 2020

Citation:

Ferrari JT and Schwartz P (2020)

Prospective Evaluation of Feline

Sourced Platelet-Rich Plasma Using

Centrifuge-Based Systems.

Front. Vet. Sci. 7:322.

doi: 10.3389/fvets.2020.00322

\section{Prospective Evaluation of Feline Sourced Platelet-Rich Plasma Using Centrifuge-Based Systems}

\author{
Jonathan T. Ferrari* and Pamela Schwartz \\ Department of Surgery, The Animal Medical Center, New York, NY, United States
}

Objective: To evaluate the hematologic components of platelet-rich plasma (PRP) generated using feline blood with two commercially available centrifuge-based systems ${ }^{1,2}$.

Materials and methods: Twenty healthy adult cats were enrolled in this prospective study from November 2018 to January 2019. Feline blood samples were obtained for analysis of whole blood (WB) cellular components and preparation of PRP product. PRP was prepared using two commercial systems and complete blood count (CBC) testing was performed on both WB and PRP samples. The cellular composition of the PRP product was compared to the WB sample for each patient.

Results: Both systems showed significant decrease of median RBC concentration in PRP products compared to WB samples ( $P=0.002$ for both systems). System 1 significantly decreased median WBC concentration $(P=0.002)$. System 2 decreased WBC concentration, though statistical significance was not reached $(P=0.63)$. Median platelet concentration was decreased by 3\% using System 1, and increased by $187 \%$ using System 2. Platelet aggregation presented a challenge with $8 / 20(40 \%)$ of samples demonstrating platelet aggregation.

Clinical relevance: Commercial systems available for generation of PRP may be useful for creating a feline sourced product and in this study showed promise in decreasing RBC and WBC concentration. Neither system tested achieved 2-5 times platelet concentration from baseline. Platelet aggregation presented a significant obstacle to reliable generation of PRP products using feline blood. This treatment modality may be particularly beneficial for feline patients with osteoarthritis and soft tissue injuries, though first characterizing the PRP product made using feline blood is critical to validate its use in further clinical studies.

Keywords: platelet-rich plasma, platelet count, feline regenerative medicine, platelet aggregation, platelet clumping

\section{INTRODUCTION}

Platelet-rich plasma (PRP) is defined as a blood concentrate with a higher concentration of platelets than whole blood (WB) (1). In addition to their hemostatic action, platelets function in wound healing by releasing alpha granules containing growth factors which initiate cellular migration, angiogenesis, and matrix deposition (2-7). Studies involving PRP use for human, equine, and

\footnotetext{
${ }^{1}$ Arthrex ACP Double Syringe System, Arthrex Orthobiologics, Naples, FL.

${ }^{2}$ Companion Regenerative Therapies Pure PRP Kit, Companion Regenerative Therapies, Fort Meyers, FL.
} 
canine patients with orthopedic, and soft tissue injuries have demonstrated improved function and perceived pain control in the settings of osteoarthritis and tendinopathies (8-18). Canine sourced PRP has also been demonstrated to improve the gross and histologic rate of wound healing, confer antibiotic properties for cutaneous wound healing, and improve survival of skin flaps (19-24). The use of PRP for management of musculoskeletal or soft tissue injuries has not been investigated in feline patients.

For a blood product to be categorized as PRP, the platelet concentration must be $>1,000 \mathrm{~K} / \mathrm{uL}$ or within a range of 2 5 times that of baseline WB concentration $(1,25)$. PRP also contains red blood cells (RBC) and white blood cells (WBC), and the ideal concentration of these components for therapeutic effect is disputed. PRP function may be enhanced by the presence of leukocytes, though some authors argue that neutrophils can induce inflammation causing tissue damage $(26,27)$. An inhibitory effect on osteoblast and fibroblast proliferation has also been described with higher platelet concentration in PRP samples (28). Thus, the variability in PRP product composition presents a challenge to reliable generation and clinical efficacy. Validation of the product composition prior to clinical use is important.

Multiple commercial systems are available for generation of PRP and prior studies have previously evaluated the cellular composition of PRP generated using canine blood (29-31). These studies show inconsistent concentrations of platelets and WBC in PRP products across commercially available systems (29-31). A recent study evaluating five different systems using canine blood found differing concentration of platelets, neutrophils, and $\mathrm{RBC}$ between systems (31). Despite the reported discrepancies in composition across systems, clinical studies using PRP in canine patients have shown benefit in osteoarthritis, wound healing, and tendinopathies (8-24).

Given the clinical benefit of PRP in human, equine, and canine patients, it is reasonable to suspect that this therapy may be similarly efficacious for feline patients. While prior reports have demonstrated success producing feline platelet concentrate (32-34), evaluation of commercially available systems has not been reported. To the authors' knowledge, the only reported use of PRP in cats is that of a canine sourced PRP applied to a cutaneous wound of a feline patient (35). Prior work has shown promise using meloxicam, tramadol, and recently a feline-specific anti-nerve growth factor monoclonal antibody in cats with osteoarthritis (36-40). However, these medical options may be limited in some feline patients. Non-steroidal antiinflammatory drugs are often contraindicated in geriatric cats with osteoarthritis due to concurrent chronic kidney disease. Some cats may develop dose-dependent side effects of tramadol use (36). Therefore, use of a feline sourced PRP may be particularly beneficial for patients with osteoarthritis and soft tissue injuries. First characterizing the PRP product made using feline blood is critical to validate its use.

The hypothesis of this study was that PRP products generated using the commercial systems would demonstrate concentration

\footnotetext{
Abbreviations: ACD-A, Anticoagulant citrate dextrose solution A; CBC, Complete blood count; EDTA, Ethylenediaminetetraacetic acid; PRP, Platelet-rich plasma; RBC, Red blood cell; WBC, White blood cell; WB, Whole blood.
}

of platelets at least two times that of WB. A secondary goal of this study was to determine the concentrations of RBC and WBC components of the PRP products in comparison to baseline WB values.

\section{MATERIALS AND METHODS}

A prospective study was performed from November 2018 through January 2019 at the Animal Medical Center Elmer and Mamdouha Bobst Hospital. In this study all feline participants were employee-owned adult cats without a history of neoplasia, anemia (hematocrit $<30 \%$ ), or concurrent use of corticosteroid or other immunosuppressive therapeutics. For inclusion in the study, cats were required to be at least 1 year of age and weigh $>3.0 \mathrm{~kg}$ to ensure safety when collecting the required blood volume. All animals were deemed healthy by veterinarian physical examination, $\mathrm{CBC}$, and serum biochemistry panel. Informed owner consent was obtained prior to inclusion in the study, and the study protocol was approved by the Institutional Animal Care and Use Committee. All patients were supervised by a veterinarian during participation in this study.

Two commercial systems were analyzed as a part of this study ${ }^{1,2}$ and patients were randomly assigned to a system. All cats were sedated to achieve a plane of sedation suitable for jugular venipuncture using dexmedetomidine ${ }^{3}$. Jugular venipuncture was performed using a sterile 21-gauge-butterfly needle for blood sample acquisition. Following sample collection, sedation was reversed using an appropriate intramuscular dose of atipamezole ${ }^{4}$. Sample collection and preparation was performed according to manufacturer recommendations, with the only modification being a limitation of the blood volume obtained to account for the lower body weight of feline patients compared to canine patients. To ensure that the blood obtained from any feline patient was $<10 \%$ of the individual's blood volume, venipuncture was restricted to $16 \mathrm{~mL}$ for System 1 and $15 \mathrm{~mL}$ for System 2. Blood samples $(2.5 \mathrm{~mL})$ were first collected for complete blood count (CBC) and placed into standard ethylenediaminetetraacetic acid (EDTA) collection tubes ${ }^{5}$, and samples were then collected for PRP generation during the same venipuncture event using manufacturer syringes. Anticoagulant citrate dextrose solution $\mathrm{A}^{6}$ (ACD-A) was added to each syringe prior to sample collection as instructed by each manufacturer.

For System 1, $1.5 \mathrm{~mL}$ of ACD-A and $13.5 \mathrm{~mL}$ of patient blood were added to the manufacturer syringe. The volumes of ACD-A and blood were within the range recommended by the manufacturer for canine patients. System 1 utilized a doublesyringe system and a single centrifugation step $(1,300 \mathrm{rpm} \times$ $5 \mathrm{~min}$ ), after which the PRP was withdrawn into the inner syringe (Figure 1A).

For System 2, $2.5 \mathrm{~mL}$ of ACD-A and $12.5 \mathrm{~mL}$ of patient blood were added to a syringe. For canine patients, the manufacturer recommended larger volumes of ACD-A and blood. In restricting

\footnotetext{
${ }^{3}$ Dexmedetomidine hydrochloride, Zoetis, Kalamazoo, MI.

${ }^{4}$ Atipamezole hydrochloride, Zoetis, Kalamazoo, MI.

${ }^{5}$ Becton, Dickinson and Company, Franklin Lakes, NJ.

${ }^{6}$ Anticoagulant citrate dextrose solution, solution A, Citra Labs, Braintree, ME.
} 


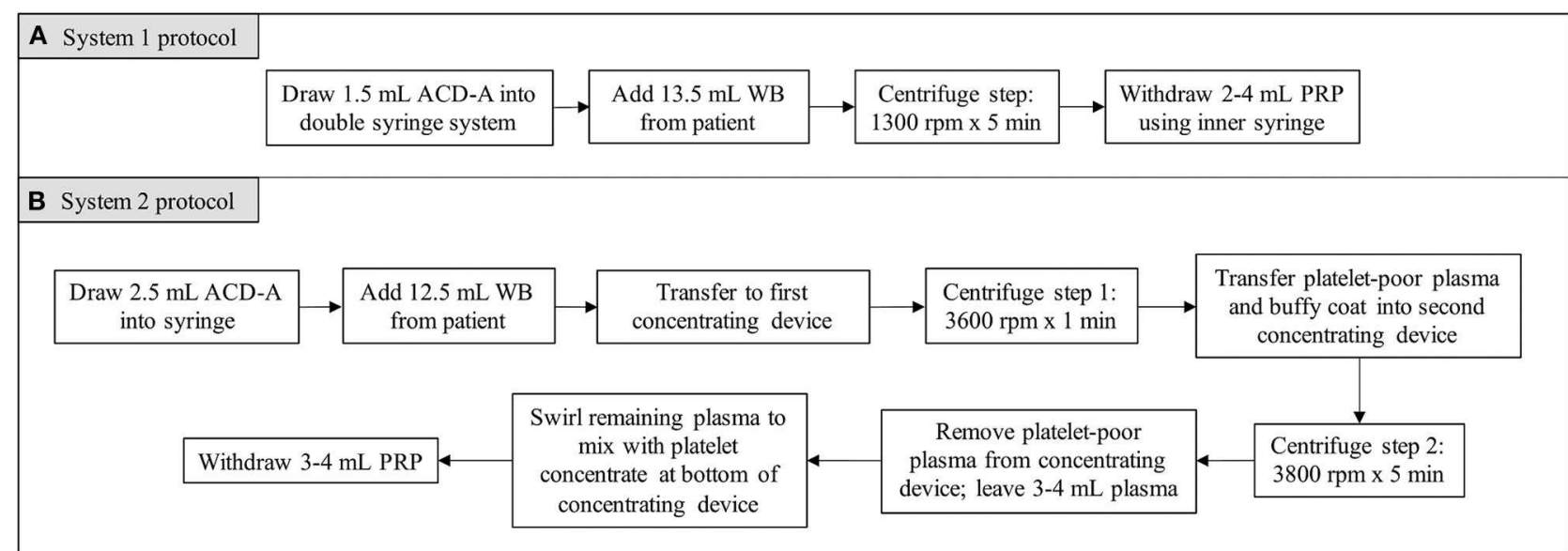

FIGURE 1 | Protocol for generation of feline PRP using System 1 (A) and System 2 (B).

the volume of blood obtained from each patient to account for the lower body weight of feline patients, the volume of ACD-A was restricted as well while maintaining the ratio recommended by the manufacturer. The blood mixed with ACD-A was transferred into the first concentrating device for the first centrifugation step $(3,600 \mathrm{rpm} \times 1 \mathrm{~min})$. Following the first centrifugation step, the platelet and plasma buffy coat was collected into a new syringe and transferred into the second concentrating device. The second centrifugation step was then performed $(3,800 \mathrm{rpm}$ $\times 5 \mathrm{~min}$ ), after which the platelet-poor plasma product was withdrawn from the concentrating device and discarded. The platelet concentrate was resuspended in the remaining plasma by gently swirling the concentrating device, and the resultant PRP was collected (Figure 1B).

Baseline CBC was performed including WBC differential, $\mathrm{RBC}$ concentration, and platelet concentration using an in-house hematology analyzer ${ }^{7}$. The same analyzer was used immediately following sample preparation to assess the PRP product. The WB and PRP product RBC, WBC, neutrophil, lymphocyte, monocyte, and platelet concentrations were determined for each sample. Data were non-normal in all except RBC (Shapiro Wilk test performed). Therefore, non-normal data were analyzed by means of the non-parametric Wilcoxon rank sum test for the unpaired comparisons of System 1-System 2 and the nonparametric Wilcoxon signed rank test for the paired comparisons of WB-PRP. Results were reported as median with lower (25th) and upper (75th) quartiles. These values were analyzed using statistical software ${ }^{8}$ and $P$-values were reported with significance set at $P<0.05$.

\section{RESULTS}

Twenty cats were enrolled in this study, with breeds represented including Domestic Shorthair $(n=18)$ and Domestic Longhair

\footnotetext{
${ }^{7}$ Idexx LaserCyte Hematology Analyzer, Idexx Laboratories, Westbrook, ME.

${ }^{8}$ SAS v. 9.3, SAS Institute Inc, Cary, NC.
}

$(n=2)$ breeds. Of these, nine were neutered males and 11 were spayed females. Mean age of patients was 5.7 years (range 1-15 years) and mean weight was $5.6 \mathrm{~kg}$ (range $3.43-8.9 \mathrm{~kg}$ ).

Ten blood samples were used for each system studied; patients were randomly assigned to a group and no patient sample was used for both groups. Group 1 was tested using System 1 and consisted of 10 Domestic Shorthair cats, and five each were female spayed and male neutered cats. The median weight of patients in group 1 was $5.35 \mathrm{~kg}$ (range $3.4-8.9 \mathrm{~kg}$ ) and the median age was 5 years (range 1-15). Group 2 was tested using System 2 and consisted of eight Domestic Shorthair and two Domestic Longhair cats, and six were female spayed and four were male neutered cats. The median weight of patients in group 2 was $5.25 \mathrm{~kg}$ (range $4.4-8.3 \mathrm{~kg}$ ) and the median age was 7 years (range 1-10).

Clumping of platelets occurred in the WB samples of $8 / 20(40 \%)$ cats; three of these samples were in the System 1 group and five were in the System 2 group. For the purposes of statistical analysis, these patients were included in analyses for concentration of RBC, neutrophils, lymphocytes, and monocytes, but excluded from statistical analysis for platelet concentration.

\section{System 1}

The volume of PRP generated using System 1 ranged from 2 to $4 \mathrm{~mL}$ per patient. Median $\mathrm{RBC}$ and $\mathrm{WBC}$ concentrations were both significantly decreased compared to the WB samples. Median RBC concentration was decreased by $99.9 \%(P=0.002)$. Median concentration of each WBC component (neutrophils, lymphocytes, monocytes) was decreased by $100 \%(P=0.002)$. Platelet clumping was documented of the WB samples for $3 / 10$ (30\%) samples. The samples with platelet clumping were excluded from analysis for platelet concentration. The median platelet concentration was decreased by $3 \%$ compared to the WB samples, and this was not statistically significant $(P=0.98)$ (Table 1). 
TABLE 1 | Median (quartile 1-quartile 3) values of cellular components of WB and PRP products generated using two commercial systems.

\begin{tabular}{lccc}
\hline & WB & PRP & P-value \\
\hline System 1 & & & \\
RBC $(\mathrm{K} / \mu \mathrm{L})$ & $8.95(9.59-8.15)$ & $0.12(0.07-0.18)$ & 0.002 \\
WBC $(\mathrm{K} / \mu \mathrm{L})$ & $9.65(8.00-10.80)$ & 0.00 & 0.002 \\
Neutrophils $(\mathrm{K} / \mu \mathrm{L})$ & $6.08(3.42-7.31)$ & 0.00 & 0.002 \\
Lymphocytes $(\mathrm{K} / \mu \mathrm{L})$ & $2.84(2.00-4.28)$ & 0.00 & 0.002 \\
Monocytes $(\mathrm{K} / \mu \mathrm{L})$ & $0.31(0.29-0.35)$ & 0.00 & 0.004 \\
Platelets $(\mathrm{K} / \mu \mathrm{L})$ & $243(193-349)$ & $238(225-297)$ & 0.98 \\
System 2 & & & 0.002 \\
RBC $(\mathrm{K} / \mu \mathrm{L})$ & $9.63(8.70-9.97)$ & $0.57(0.28-1.78)$ & 0.92 \\
WBC $(\mathrm{K} / \mu \mathrm{L})$ & $6.95(6.50-8.60)$ & $1.35(0.40-15.10)$ & 0.63 \\
Neutrophils $(\mathrm{K} / \mu \mathrm{L})$ & $4.47(3.71-5.45)$ & $0.60(0.16-6.54)$ & 0.63 \\
Lymphocytes $(\mathrm{K} / \mu \mathrm{L})$ & $2.09(1.80-2.23)$ & $0.60(0.19-6.93)$ & 0.63 \\
Monocytes $(\mathrm{K} / \mu \mathrm{L})$ & $0.24(0.20-0.28)$ & $0.08(0.02-1.06)$ & 0.44 \\
Platelets $(\mathrm{K} / \mu \mathrm{L})$ & $269(239-291)$ & $505(166-1094)$ & \\
\hline
\end{tabular}

$N=10$ for all, except for platelet analysis, for which $n=7$ for System 1 and $n=5$ for System 2 due to sample exclusion when affected by platelet clumping detected in the WB samples. Comparisons were paired non-parametric by means of the Wilcoxon signed rank test (WSRT). $P$-values were reported with significance set at $P<0.05$.

\section{System 2}

The volume of PRP made using System 2 ranged from 3 to $4 \mathrm{~mL}$ per patient. Median RBC concentration was significantly decreased compared to the WB samples by $94 \%(P=0.002)$. Median WBC concentration was decreased by $80 \%$ and this difference was not statistically significant $(P=0.92)$. When comparing the PRP product to the WB samples, neutrophil concentration was decreased by $86.6 \%(P=0.63)$, lymphocyte concentration was decreased by $71 \%(P=0.63)$, and monocyte concentration was decreased by $66.6 \%(P=0.63)$. Platelet clumping was documented of the WB samples for $5 / 10$ (50\%) of the samples. The samples with platelet clumping were excluded from analysis for platelet concentration. The median platelet concentration of the included PRP products was increased by $187 \%$ compared to the WB samples, and this was not statistically significant $(P=0.44)$ (Table 1$)$.

\section{DISCUSSION}

In this study, both systems reduced the median RBC concentration of the PRP products compared to WB samples. However, neither system concentrated platelets by $2-5$ times baseline, or had platelet concentration $>1,000 \mathrm{~K} / \mathrm{uL}$, which is ideal for a PRP product $(1,25)$. Median platelet concentration was decreased by 3\% using System 1, while System 2 increased the median platelet concentration by $187 \%$. Therefore, the use of the term "PRP" to describe these products may be inappropriate. For the purpose of consistency and clarity, this discussion will continue to use the term "PRP," though the validity of characterizing these products as such is in question.

System 1 completely excluded monocytes, neutrophils, and lymphocytes. However, this system did not result in an increase in median platelet concentration. System 1 required only one step of centrifugation, eliminating a second step of centrifugation during preparation that is common in other PRP systems. It is possible that for feline blood, the second centrifugation step may important for platelet concentration, or that centrifuge settings should be altered as those used in this study were those recommended for canine blood. This may represent a species difference between the feline samples tested in this study and canine blood samples for which this system was manufactured. As this study did not assess the clinical efficacy of these products, it is unknown if the PRP products generated using System 1, which had similar platelet concentrations to the WB samples with significantly reduced $\mathrm{RBC}$ and $\mathrm{WBC}$ concentration, would have applications in vivo.

System 2 increased median platelet concentration in the PRP samples ( $187 \%$ increase), though this concentrate falls short of the ideal concentration 2-5 times the baseline. Several samples $(5 / 10)$ were excluded due to platelet clumping detected in the $\mathrm{WB}$, perhaps resulting in type II error. It is possible that the median platelet concentration may be higher if fewer samples demonstrated platelet clumping and could have been included in analysis. System 2 demonstrated $80 \%$ reduction of leukocyte concentration, which characterizes this product as leukocyte-poor PRP. This data demonstrating nearly 2 -fold platelet concentration is promising for the potential clinical use of this product.

A major finding of this study was platelet clumping documented in $8 / 20(40 \%)$ WB samples. The samples that clumped were excluded from statistical analysis for platelet concentration, which resulted in smaller sample sizes. Platelet aggregation is a common problem resulting in pseudothrombocytopenia in feline hematologic laboratory samples (32). As platelet aggregation occurred in both groups, it is suspected that platelet aggregation was due to species-specific factors or collection method. In the present study, WB samples for $\mathrm{CBC}$ processing were collected first and placed within standard EDTA-collection tubes, followed by blood collection into syringes for PRP preparation with ACD-A pre-loaded in the syringe as anticoagulant. The anticoagulant used here (EDTA for WB sample for baseline CBC; ACD-A for PRP product preparation) may not be appropriate for feline blood in this application. It has been previously suggested that EDTA rather than citrate should be used for anticoagulation of canine samples, and other anticoagulants including iloprost have been recommended for feline blood samples (34, 41, 42). Another consideration may be applying ACD-A to the sterile line used for venipuncture, thereby admixing the WB samples with anticoagulant within the line prior to entry into the tube or syringe. Further investigation is warranted to determine the best method to prevent feline platelet aggregation.

This study was limited by small sample size, which may have resulted in type II error. Manual platelet counting was not included in this study and may be a method to avoid eliminating samples due to platelet aggregation affecting instrument counting. In addition, the method of feline platelet counting was not validated and it has been suggested that hematologic analyzers be validated specifically for PRP samples to ensure accurate platelet counting (43).

The study reported here was designed to evaluate the ability of commercial systems to concentrate platelets using feline blood, 
as well as to evaluate the other cellular components of the PRP products. The data show that the cellular composition of PRP products from the systems differed in important ways. System 1 did not result in an increased concentration of platelets. System 2 showed an increase in the median platelet concentration, though not to a degree to meet the standard definition of PRP. These results underscore the need for standardization and validation of these systems if clinical efficacy is to be expected. Conclusions regarding the clinical use of the PRP products cannot be drawn from this study. Further study is necessary for evaluation of clinical efficacy of PRP use in feline patients.

\section{DATA AVAILABILITY STATEMENT}

The datasets generated for this study are available on request to the corresponding author.

\section{REFERENCES}

1. Saunders WB, Bearden RN, Franklin SP. Platelet-rich plasma and autologous conditioned sera. In: Johnston SA, Tobias KM, editors. Veterinary Surgery: Small Animal. 2nd Edn. St. Louis: Elsevier Inc. (2018) 40-8.

2. Boswell SG, Cole BJ, Sundman EA, Karas V, Fortier LA. Platelet-rich plasma: a milieu of bioactive factors. Arthroscopy. (2012) 28:429-39. doi: 10.1016/j.arthro.2011.10.018

3. Filardo G, Kon E, Roffi A, Di Matteo B, Merli ML, Marcacci M. Platelet rich plasma: why intra-articular? a systematic review of preclinical studies and clinical evidence on PRP for joint degeneration. Knee Surg Sports Traumatol Arthrosc. (2015) 23:2459-74. doi: 10.1007/s00167-013-2743-1

4. Hsu WK, Mishra A, Rodeo SR, Fu F, Terry MA, Randelli P, et al. Platelet-rich plasma in orthopaedic applications: evidence-based recommendations for treatment. J Am Acad Orthop Surg. (2013) 21:739-48. doi: 10.5435/00124635-201312000-00004

5. McLellan J, Plevin S. Does it matter which platelet-rich plasma we use? Equine Vet Educ. (2011) 23:101-4. doi: 10.1111/j.2042-3292.2010. 00185.x

6. Pelletier MH, Malhotra A, Brighton T, Walsh WR, Lindeman R. Platelet function and constituents of platelet rich plasma. Int J Sports Med. (2013) 34:74-80. doi: 10.1055/s-0032-1316319

7. Sundman EA, Cole BJ, Karas V, Della Valle C, Tetreault MW, Mohammed $\mathrm{HO}$, et al. The anti-inflammatory and matrix restorative mechanisms of platelet-rich plasma in osteoarthritis. Am J Sports Med. (2014) 42:35-41. doi: 10.1177/0363546513507766

8. Sampson S, Gerhardt M, Mandelbaum B. Platelet rich plasma injection grafts for musculoskeletal injuries: a review. Curr Rev Musculoskelet Med. (2008) 1:165-74. doi: 10.1007/s12178-008-9032-5

9. Franklin S, Cook J. Prospective trial of autologous conditioned plasma versus hyaluronan plus corticosteroid for elbow osteoarthritis in dogs. Can Vet J. (2013) 54:881-4.

10. Silva RF, Carmona JU, Rezende CM. Intra-articular injections of autologous platelet concentrates in dogs with surgical reparation of cranial cruciate ligament rupture. Vet Comp Orthop Traumatol. (2013) 26:285-90. doi: 10.3415/VCOT-12-06-0075

11. Smith JJ, Ross MW, Smith RK. Anabolic effects of acellular bone marrow, platelet rich plasma, and serum on equine suspensory ligament fibroblasts in vitro. Vet Comp Orthop Traumatol. (2006) 19:43-7. doi: 10.1055/s-0038-1632972

12. Patel S, Dhillon MS, Aggarwal S, Marwaha N, Jain A. Treatment with platelet-rich plasma is more effective than placebo for knee osteoarthritis: a prospective, double-blinded, randomized trial. Am J Sports Med. (2013) 41:356-64. doi: 10.1177/0363546512471299

\section{ETHICS STATEMENT}

The animal study was reviewed and approved by Institutional Animal Care and Use Committee of the Animal Medical Center. Written informed consent was obtained from the owners for the participation of their animals in this study.

\section{AUTHOR CONTRIBUTIONS}

Both authors assisted with study development, procedures, and manuscript preparation.

\section{ACKNOWLEDGMENTS}

Statistical analysis was performed by Joe Hauptman, DVM, MS, DACVS-SA.

13. Raeissadat SA, Rayegani SM, Babaee M, Ghorbani E. The effect of platelet rich plasma on pain, function, and quality of life of patients with knee osteoarthritis. Pain Res Treat. (2013) 2013:165967. doi: 10.1155/2013/165967

14. Ho LK, Baltzer WI, Nemanic S, Stieger-Vanegas SM. Single ultrasound-guided platelet-rich plasma injection for treatment of supraspinatus tendinopathy in dogs. Can Vet J. (2015) 56:845-9.

15. Canapp SO, Canapp DA, Ibrahim V, Carr BJ, Cox C, Barrett JG et al. The use of adipose-derived progenitor cells and platelet-rich plasma combination for the treatment of supraspinatus tendinopathy in 55 dogs: a retrospective study. Front Vet Sci. (2016) 3:61. doi: 10.3389/fvets.2016.00061

16. Fahie MA, Ortolano GA, Guercio V, Schaffer JA, Johnston G, Au J, et al. A randomized controlled trial of the efficacy of autologous platelet therapy for the treatment of osteoarthritis in dogs. J Am Vet Med Assoc. (2013) 243:1291-7. doi: 10.2460/javma.243.9.1291

17. Cook JL, Smith PA, Bozynzki CC, Kuroki K, Cook CR, Stoker AM, et al. Multiple injections of leukoreduced platelet rich plasma reduce pain and functional impairment in a canine model of ACL and meniscal deficiency. $J$ Orthop Res. (2016) 34:607-15. doi: 10.1002/jor.23054

18. Upchurch DA, Renberg WC, Roush JK, Milliken GA, Weiss ML. Effects of administration of adipose-derived stromal vascular fraction and platelet-rich plasma to dogs with osteoarthritis of the hip joints. Am J Vet Res. (2016) 77:940-51. doi: 10.2460/ajvr.77.9.940

19. Jee CH, Eom NY, Jang HM, Choi ES, Won JH, Hong IH, et al. Effect of autologous platelet-rich plasma application on cutaneous wound healing in dogs. J Vet Sci. (2016) 17:79-87. doi: 10.4142/jvs.2016.17.1.79

20. Kim JH, Park C, Park HM. Curative effect of autologous platelet-rich plasma on a large cutaneous lesion in a dog. Vet Dermatol. (2009) 20:123-6. doi: 10.1111/j.1365-3164.2008.00711.x

21. Farghali HA, AbdElKader NA, AbuBakr HO, Aljuaydi SH, Khattab MS, Elhelw R, et al. Antimicrobial action of autologous platelet-rich plasma on MRSA-infected skin wounds in dogs. Sci Rep. (2019) 9:12722. doi: 10.1038/s41598-019-48657-5

22. Farghali HA, AbdElKader NA, Khattab MS, AbuBakr HO. Evaluation of subcutaneous infiltration of autologous platelet-rich plasma on skin-wound healing in dogs. Biosci Rep. (2017) 37:BSR20160503. doi: 10.1042/BSR20160503

23. Karayannopoulou M, Papazoglou LG, Loukopoulos P, Kazakos G, Chantes A, Giannakas N, et al. Locally injected autologous platelet-rich plasma enhanced tissue perfusion and improved survival of long subdermal plexus skin flaps in dogs. Vet Comp Orthop Traumatol. (2014) 27:379-86. doi: 10.3415/VCOT-14-02-0030

24. Karayannopoulou M, Psalla D, Kazakos G, Loukopoulos P, Giannakas $\mathrm{N}$, Savvas I, et al. Effect of locally injected autologous platelet-rich plasma on second intention wound healing of acute full-thickness 
skin defects in dogs. Vet Comp Orthop Traumatol. (2015) 28:172-8. doi: 10.3415/VCOT-14-06-0088

25. Marx RE. Platelet-rich plasma (PRP): what is PRP and what is not PRP? Implant Dent. (2001) 10:225-8. doi: 10.1097/00008505-200110000-00002

26. Andia I, Sanchez M, Maffulli N. Basic science: molecular and biological aspects of platelet-rich plasma therapies. Oper Tech Orthop. (2012) 22:3-9. doi: 10.1053/j.oto.2011.09.005

27. Sundman EA, Cole BJ, Fortier LA. Growth factor and catabolic cytokine concentrations are influenced by the cellular composition of platelet-rich plasma. Am J Sports Med. (2011) 39:2135-40. doi: 10.1177/036354651 1417792

28. Graziani F, Ivanovski S, Cei S, Ducci F, Tonetti M, Gabriele M. The in vitro effect of different PRP concentrations on osteoblasts and fibroblasts. Clin Oral Implants Res. (2006) 17:212-9. doi: 10.1111/j.1600-0501.2005.01203.x

29. Stief M, Gottschalk J, Ionita JC, Eispanier A, Oechtering H, Böttcher P. Concentration of platelets and growth factors in canine autologous conditioned plasma. Vet Comp Orthop Traumatol. (2011) 24:122-5. doi: 10.3415/VCOT-10-04-0064

30. Franklin SP, Garner BC, Cook JL. Characteristics of canine platelet-rich plasma prepared with five commercially available systems. Am J Vet Res. (2015) 76:822-7. doi: 10.2460/ajvr.76.9.822

31. Carr BJ, Canapp SO, Mason DR, Cox C, Hess T. Canine plateletrich plasma systems: a prospective analysis. Front Vet Sci. (2016) 2:73. doi: 10.3389 /fvets.2015.00073

32. Silva RF, Alvarez ME, Ríos DL, López C, Carmona JU, Rezende CM. Evaluation of the effect of calcium gluconate and bovine thrombin on the temporal release of transforming growth factor beta 1 and platelet-derived growth factor isoform BB from feline platelet concentrates. BMC Vet Res. (2012) 8:212. doi: 10.1186/1746-6148-8-212

33. Silva RF, Carmona JU, Rezende CM. Ultrastructural characteristics of fibrin clots from canine and feline platelet concentrates activated with calcium gluconate or calcium gluconate plus batroxobin. BMC Vet Res. (2013) 9:77. doi: 10.1186/1746-6148-9-77

34. Riond B, Waßmuth AK, Hartnack S, Hofmann-Lehmann R, Lutz H. Effective prevention of pseudothrombocytopenia in feline blood samples with the prostaglandin $I_{2}$ analogue iloprost. BMC Vet Res. (2015) 11:183. doi: 10.1186/s12917-015-0510-x

35. Gemignani F, Perazzi A, Iacopetti I. Use of canine sourced platelet-rich plasma in a feline contaminated cutaneous wound. Can Vet J. (2017) 58:141-4.

36. Guedes AGP, Meadows JM, Pyendop BH, Johnson EG. Evaluation of tramadol for treatment of osteoarthritis in geriatric cats. J Am Vet Med Assoc. (2018) 252:565-71. doi: 10.2460/javma.252.5.565
37. Gruen ME, Thomson AE, Griffith EH, Paradise H, Gearing DP, Lascelles BD. A feline-specific anti-nerve growth factor antibody improves mobility in cats with degenerative joint disease-associated pain: a pilot proof of concept study. J Vet Intern Med. (2016) 30:1138-48. doi: 10.1111/jvim.13972

38. Lascelles BD, Hansen BD, Roe S, DePuy V, Thomson A, Pierce CC, et al. Evaluation of client-specific outcome measures and activity monitoring to measure pain relief in cats with osteoarthritis. J Vet Intern Med. (2007) 21:410-6. doi: 10.1111/j.1939-1676.2007.tb02983.x

39. Gruen ME, Griffith E, Thomson A, Simpson W, Lascelles BD. Detection of clinically relevant pain relief in cats with degenerative joint disease associated pain. J Vet Intern Med. (2014) 28:346-50. doi: 10.1111/jvim.12312

40. Gruen ME, Griffith EH, Thomson AE, Simpson W, Lascelles BD. Criterion validation testing of clinical metrology instruments for measuring degenerative joint disease associated mobility impairment in cats. PLOS ONE. (2015) 10:e0131839. doi: 10.1371/journal.pone.0131839

41. Stokol T, Erb HN. A comparison of platelet parameters in EDTA- and citrate-anticoagulated blood in dogs. Vet Clin Pathol. (2007) 36:148-54. doi: 10.1111/j.1939-165X.2007.tb00201.x

42. Mylonakis ME, Leontides L, Farmaki R, Kostoulas P, Koutinas AF, Christopher M. Effect of anticoagulant and storage conditions on platelet size and clumping in healthy dogs. J Vet Diagn Invest. (2008) 20:774-9. doi: $10.1177 / 104063870802000609$

43. Woodell-May JE, Ridderman DN, Swift MJ, Higgins J. Producing accurate platelet counts for platelet rich plasma: validation of a hematology analyzer and preparation techniques for counting. J Craniofac Surg. (2005) 16:749-56. doi: 10.1097/01.scs.0000180007.30115.fa

Conflict of Interest: Centrifuge and regenerative medicine products were provided by Arthrex ${ }^{1}$ for the purposes of this study. No other third-party funding or support was received in connection with study design, testing, data analysis, or manuscript preparation.

The authors declare that the research was conducted in the absence of any commercial or financial relationships that could be construed as a potential conflict of interest.

Copyright $\odot 2020$ Ferrari and Schwartz. This is an open-access article distributed under the terms of the Creative Commons Attribution License (CC BY). The use, distribution or reproduction in other forums is permitted, provided the original author(s) and the copyright owner(s) are credited and that the original publication in this journal is cited, in accordance with accepted academic practice. No use, distribution or reproduction is permitted which does not comply with these terms. 